\title{
Experimental analysis on temperature characteristics of a rectangular bulk YBCO exposed to alternating magnetic field
}

\author{
Peng Pang ${ }^{1} \mathbb{D} \cdot$ Wei Liu ${ }^{1} \cdot$ Xiaochen Ren $^{1} \cdot$ Qinlong Lu $^{2} \cdot$ Shihong Yang ${ }^{2} \cdot$ Hailian Jing ${ }^{1} \cdot$ Xingyu Zhou $^{1}$
}

(c) Springer Nature Switzerland AG 2019

\begin{abstract}
The motion of magnetic flux will cause temperature rise inside of a bulk high-temperature superconductor (HTS) when exposed to an alternating magnetic field (AMF). In levitation system, like superconducting motors, bearing and vehicle, however, bulk HTSs are always subjected to ripple fields due to mechanical vibration, as well as harmonic field of armature windings, which will cause energy loss inside of bulk HTSs. Part of the heat caused by the energy loss is transferred to liquid nitrogen $\left(\mathrm{LN}_{2}\right)$ through the surface of bulk $\mathrm{HTSs}$, and the other part will lead to temperature rise inside bulk HTSs. We experimentally analyzed the temperature characteristics of bulk YBCO under different AMF frequencies in this article. And the effect of AMF on the temperature rise of the bulk HTS under different cooling heights (CHs) and working heights (WHs) was also studied. The results showed that the higher the $\mathrm{CH}$, the greater the temperature rises inside the bulk HTS. Besides, the temperature of the whole bulk HTS increases with the decrease in the WH and the increase in AMF frequency. The results and analyses of this article are useful for the application of bulk HTSs in the high-speed rotating and transportation systems.
\end{abstract}

Keywords Temperature rise · Alternating magnetic field $\cdot$ High-temperature superconductor $\cdot$ Energy loss $\cdot$ Levitation system

\section{Introduction}

The high-speed levitation system based on permanent magnets (PMs) and bulk high-temperature superconductors (HTSs) has demonstrated the tremendous potentials in several fascinating applications, such as superconducting maglev vehicles [1-5], linear synchronous motors [6-8], and superconducting flywheel energy storage systems [9-11]. Because of its competitive features like self-stability without energy input, being free from mechanical friction, and no need for active control system, research on levitation force and guiding force of superconducting levitation system has been studied $[12,13]$, and however, behaviors of bulk HTSs in an alternating magnetic field (AMF) will affect the performance of the superconducting levitation system. Therefore, it is necessary to study the characteristics of bulk HTSs exposed to an AMF. The current decay characteristics of an HTS coil operated in persistent current mode under AMF are widely studied [14-16], which could be instructive for reduction in current decay of HTS coils. The loss of suspension force of a bulk HTS in the AMF above a PM guide rail has been studied, and results showed that the decrease in the guiding force became more obvious with the increase in the amplitude of the magnetic field [17]. Nevertheless, to the best of our knowledge, studies on temperature effects of bulk HTSs in the case that bulk HTSs are exposed to AMF have not been widely reported and are still only in theory. The heat generated in the bulk HTS will cause temperature rise, which directly reduces the critical current density and increases the penetration area of the external AMF $[18,19]$. So it is

Peng Pang, 761014631@qq.com | ${ }^{1}$ Chengdu University of Technology, Chengdu 610059, China. ${ }^{2}$ The 16 th Institute of China Electronic Technology Group Corporation, Hefei 230043, China.

SN Applied Sciences (2019) 1:749 | https://doi.org/10.1007/s42452-019-0745-4 


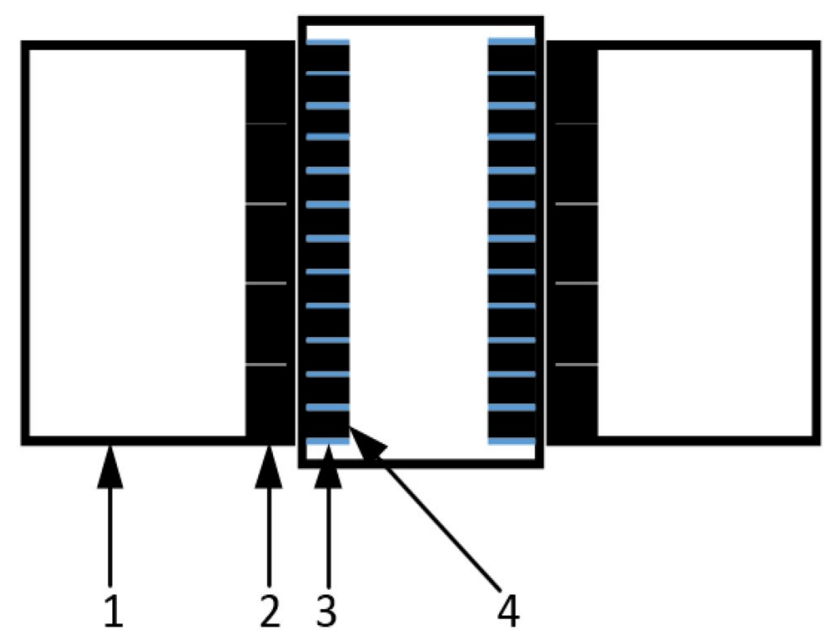

Fig. 1 Cross-sectional view of the HTS bearing (1. copper ring, 2. bulk HTS array, 3. iron ring, 4. PM ring.)

also necessary to study the temperature characteristics of bulk HTSs under a high-gradient AMF.

We are now designing a high-capacity HTS flywheel used for energy storage in a subway station. Cross-sectional view of an HTS bearing used in an HTS flywheel is shown in Fig. 1. Rotor of the HTS bearing is composed of iron rings (3) and PM rings (4), which provides magnetic field. The rotor is mounted on the shaft of the flywheel rotor. Bulk HTSs (2) are fixed in the copper ring (1), which constitute a stator of the HTS bearing. Structure of the HTS flywheel can be referred [20]. Rotor of the flywheel system can be levitated once it is moved up and down due to the flux pinning effect of bulk HTSs. To make the flywheel system and superconducting Maglevevacuated tube transport system commercially available [21], it was significant to know the working condition of bulk HTSs when they experience an AMF, which would be encountered when the PMs break locally or periodically. Because when the flywheel works in subway station, the vibration of the train, the earthquake or the rupture of the PM rotor would occur, it would cause an AMF. Besides, an AMF also exists in an HTS synchronous motor when the bulk HTS rotor and stator are not synchronized and the rotor will suffer rotating magnetic field, with structure as in [22]. The purpose of this paper is to verify the stability and reliability of the HTS flywheel in such cases and whether the flywheel can reach the purpose of commercial application.

To investigate temperature characteristics of bulk HTSs exposed to an AMF. A testing platform was designed to simulate such a magnetic field. We study the temperature characteristics of the bulk HTS and qualitatively analyze the experimental results.

\section{Experimental setup}

\subsection{Experimental details}

Figure 2 exhibits the high-speed test device. The device is mainly composed of nine components, which include a base (1) to support the device, a high-speed drive motor coupled with a PM turntable (2) to provide AMF, a cryogenic vessel used for storing liquid nitrogen $\left(\mathrm{LN}_{2}\right)$, a wall frame (4) to support the bulk HTS, a horizontal sliding rail (5), and a vertical sliding rail (6) to adjust the position of the bulk HTS. It is worth mentioning that the cryogenic vessel is covered with an aluminum plate-protective cover (3) to protect people and equipment from magnetic particles. In addition, a data transmission line (8) is used to convey the temperature signals of the bulk HTS to the control cabinet (9), where the temperature data can be recorded. It is worth mentioning that the temperature signals can also be manually recorded through a temperature controller. In this experiment, since the temperature in the center of the bulk HTS changes violently and the software cannot record values in time. So the instantaneous temperatures are manually recorded at even intervals of $20 \mathrm{~s}$.

Figure 3 depicts the PM turntable and the cryogenic vessel in detail. PMs are nested in a turntable, which is driven by a high-speed centrifugal motor below. The bottom thickness of the cryogenic vessel is $1 \mathrm{~mm}$. The bulk HTS is fixed above the bottom of the cryogenic vessel. The whole system was mounted on ground so that the system would not be shaken by Lorentz force. Accelerating the turntable from 0 to the target speed, the period is set as

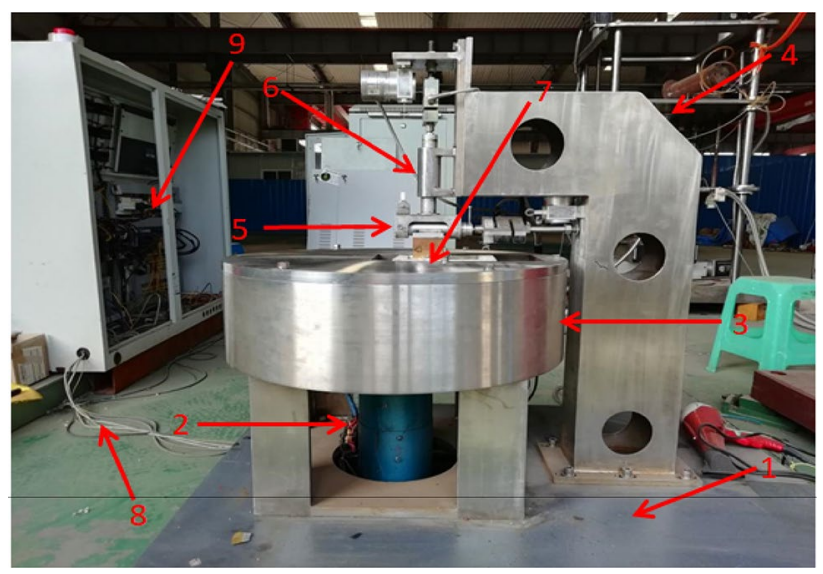

Fig. 2 The experimental system, composed of (1) the base, (2) the high-speed driving motor and PM turntable, (3) the protective cover, (4) the wall frame, (5) the horizontal sliding rail, (6) the vertical sliding rail, (7) the bulk HTS holding and temperature detection device (the detailed structure is shown in Fig. 3), (8) the data transmission line, and (9) the control cabinet 


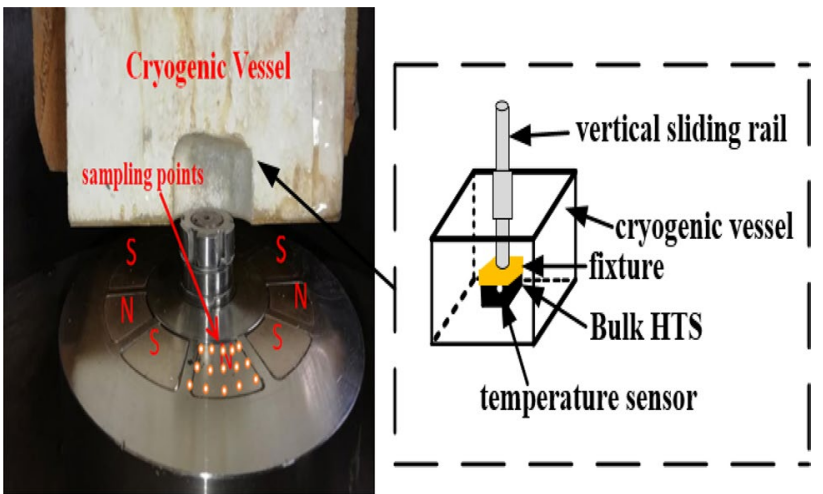

Fig. 3 The PM turntable and the cryogenic vessel

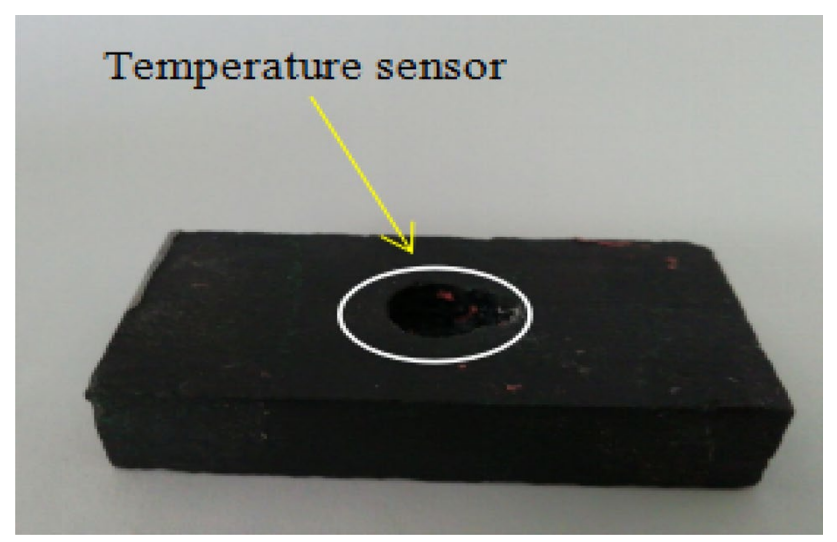

Fig. 4 The YBCO bulk sample

$2 \mathrm{~s}$. The PMs are arranged with alternating magnetic poles. To make results more accurate, we choose 15 sampling points on the surface of each PM. Every 5 points located on the inner side, the middle side, and the outer side of the PM. To display the distribution of magnetic flux density (MFD) above the PM turntable, we, respectively, measured the magnitude of MFD at 2, 4, 6, and $8 \mathrm{~mm}$ above each PM by Gauss gauge. Then, we plotted the distribution of MFD when the turntable rotates along the inner, the middle, and the outer sides of working surface (every 50 sampling points in a line), respectively.

The bulk HTS sample used in the experiment is a rectangular three-seeded crystal YBCO bulk with $64 \mathrm{~mm}$ in length, $32 \mathrm{~mm}$ in width, and $12 \mathrm{~mm}$ in height, as displayed in Fig. 4. A hole is drilled at the center of the bulk HTS surface to locate the temperature sensor (it is dangerous to punch holes on the bulk HTS. In the practical application of the bulk HTS, it will affect the performance of the bulk HTS). The PT100 temperature sensor is used to measure the temperature inside of the bulk HTS, and temperature variable was converted in terms of standardized output signal. The temperature range of PT100 is $-200{ }^{\circ} \mathrm{C}$

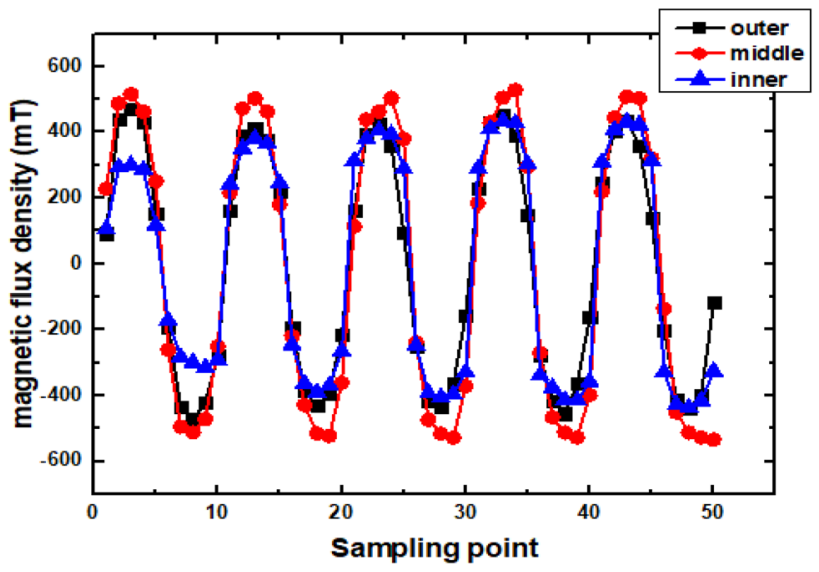

Fig. 5 MFD on the surface of $2 \mathrm{~mm}$ away from the PM surface

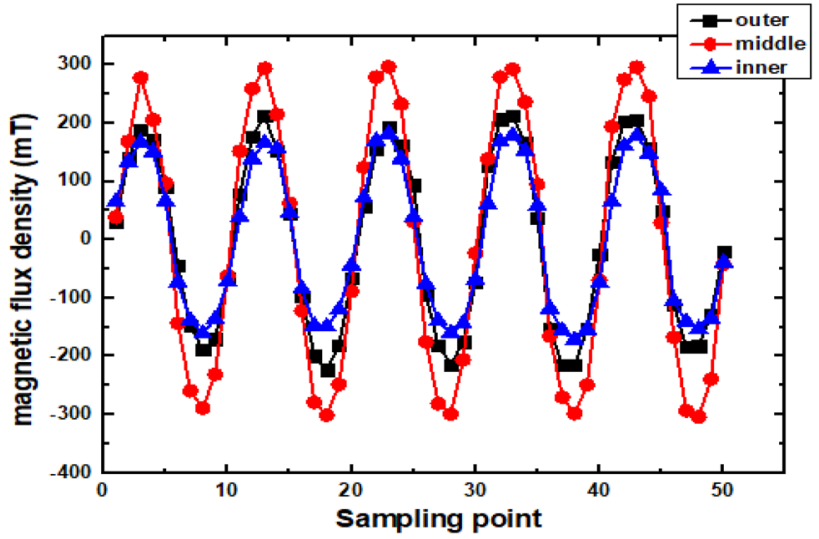

Fig. 6 MFD on the surface of $8 \mathrm{~mm}$ away from the PM surface

to $+800^{\circ} \mathrm{C}$. It is further noted that the temperature sensor is sealed at the hole to prevent heat leakage.

\subsection{Experimental process}

Here, we show the distribution of MFD on the surfaces that are $2 \mathrm{~mm}$ and $8 \mathrm{~mm}$ away from the PM turntable in Figs. 5 and 6, respectively. From Figs. 5 and 6, we can see that the distribution of MFD above the PM turntable is sinusoidal. Besides, the MFD at the middle points is higher than that in both the inner points and the outer points. The purpose of building this kind of MFD distribution is to simulate that the bulk HTS is exposed to the AMF. We used points instead of time as the $x$-axis because the MFD at the same position at different times is different during the experiment.

Before the flywheel operates, the PM rotor needs to be cooled at a certain height, namely the cooling height (CH) [23]. We firstly tried to study the influence of $\mathrm{CH}$ on the temperature characteristics of the bulk HTS under 
different rotating speeds of PM turntable. The bulk HTS was first cooled in cryogenic vessel filled with $\mathrm{LN}_{2}$ for $15 \mathrm{~min}$ to the superconducting state at $20 \mathrm{~mm}$ and $10 \mathrm{~mm}$ away from the surface of PM turntable, respectively. Here, the $10 \mathrm{~mm}$ and $20 \mathrm{~mm}$ are the $\mathrm{CH}$. Then, the bulk HTS has moved to $2 \mathrm{~mm}$ (working height (WH)) away from the PM surface by vertical sliding rail and stayed there for $5 \mathrm{~min}$. After that, we adjusted the speed of PM turntable to $1000 \mathrm{rpm}$ and kept the PM turntable rotating for $5 \mathrm{~min}$. Meanwhile, we recorded the temperature data of the bulk HTS. After measuring, the motor was turned off and the bulk HTS moved away from the PM surface. After the bulk HTS became normal, we repeated the above steps and adjusted the motor speed to $3000 \mathrm{rpm}, 5000 \mathrm{rpm}$, and $9000 \mathrm{rpm}$. Here, it should be noted that after testing, we will adjust the turntable to the initial position.

Moreover, we adjusted the WH of the bulk HTS as $4 \mathrm{~mm}$ and $6 \mathrm{~mm}$, respectively, to study the influence of WH on the temperature characteristics of the bulk HTS and obtained the temperature data in the cases that the speed changes from $1000 \mathrm{rpm}$ to $9000 \mathrm{rpm}$.

When the $\mathrm{CH}$ and WH of the bulk HTS are determined, we further studied the influence of rotating speed (1000 rpm, $3000 \mathrm{rpm}, 5000 \mathrm{rpm}$, and $9000 \mathrm{rpm}$ ) on the temperature characteristics of the bulk HTS.

Note that the copper-made temperature sensor would induce eddy current in the AMF, and its own temperature will increase. So, we considered the effect of the temperature rise of the sensor itself and rectified the data by contrast experiments in the data processing.

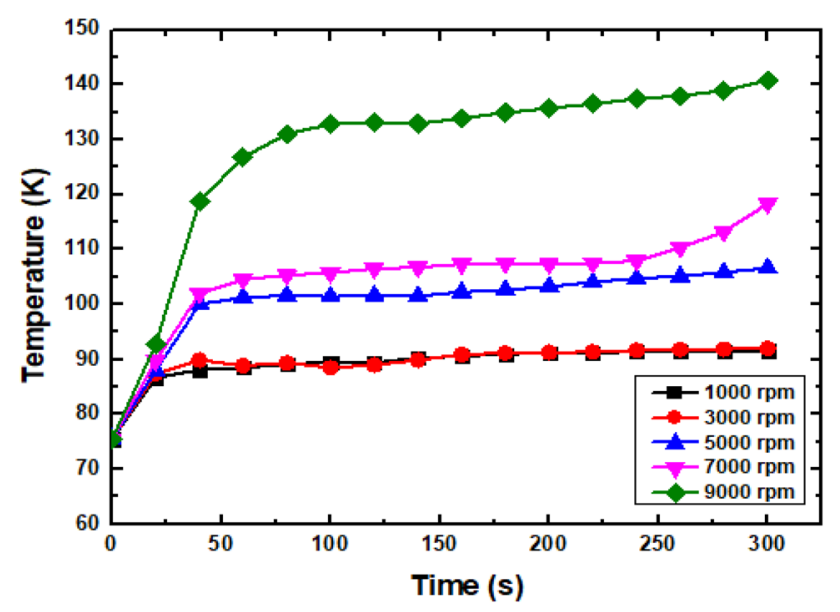

Fig. 7 Variation of temperature with time under different rotating speeds of PM turntable in the case that the $\mathrm{CH}$ is $10 \mathrm{~mm}$ and the WH is $4 \mathrm{~mm}$

\section{Results and discussion}

\subsection{Influence of $\mathrm{CH}$ on temperature characteristics}

Figures 7 and 8 plot the temperature curves under different rotating speeds of PM turntable in the cases that the WH is $4 \mathrm{~mm}$ and the $\mathrm{CH}$ is $10 \mathrm{~mm}$ and $20 \mathrm{~mm}$.

By comparing Figs. 7 and 8, we found the temperature rise in the case that $\mathrm{CH}$ is $10 \mathrm{~mm}$ is lower than that of $20 \mathrm{~mm}$ under the same rotating speed of PM turntable. Table 1 also lists the temperature values at different times at $5000 \mathrm{rpm}$. We can see that the temperature during the $10-4-\mathrm{mm}$ process is lower than that during the $20-4-\mathrm{mm}$ process. This is because less magnetic flux was captured due to the shorter travel distance at 10-4 $\mathrm{mm}$. As analyzed above, the lower the $\mathrm{CH}$, the less internal loss of the bulk HTS at the same WH. The "10-4 mm" and

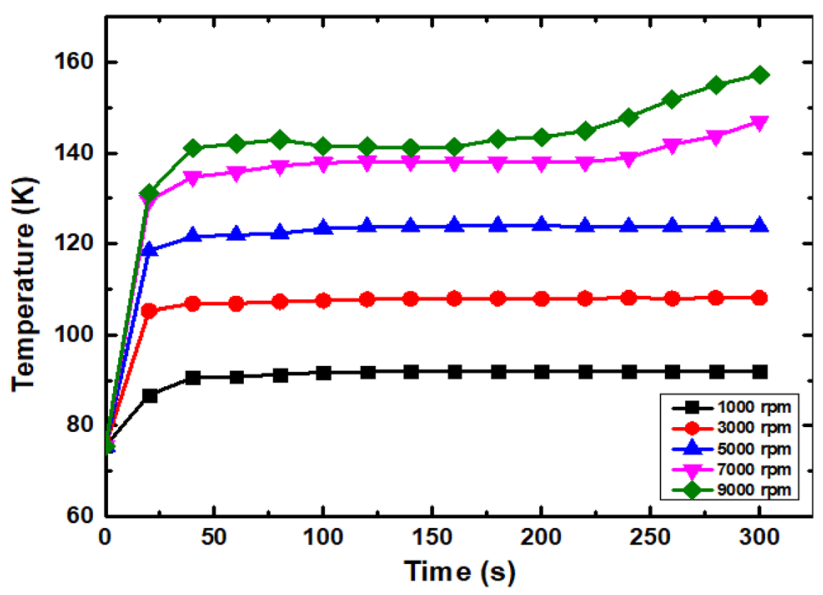

Fig. 8 Variation of temperature with time under different rotating speeds of PM turntable in the case that the $\mathrm{CH}$ is $20 \mathrm{~mm}$ and the $\mathrm{WH}$ is $4 \mathrm{~mm}$

Table 1 Temperature values at different times at $5000 \mathrm{rpm}$

\begin{tabular}{lll}
\hline Time (s) & $\begin{array}{l}\text { Temperature under } \\
10-4 \mathrm{~mm}(\mathrm{~K})\end{array}$ & $\begin{array}{l}\text { Temperature } \\
\text { under 20-4 mm } \\
(\mathrm{K})\end{array}$ \\
\hline 0 & 77 & 77 \\
20 & 88 & 116 \\
40 & 100 & 125 \\
60 & 101 & 125 \\
80 & 101 & 125 \\
100 & 101 & 125 \\
200 & 103 & 126 \\
300 & 106 & 126 \\
\hline
\end{tabular}


"20-4 $\mathrm{mm}$ " are used here: The first number represents $\mathrm{CH}$, and the second number indicates $\mathrm{WH}$.

Moreover, we noticed that temperature curves are almost coincident under the rotating speed of $1000 \mathrm{rpm}$ and $3000 \mathrm{rpm}$ of PM turntable in Fig. 7. However, the temperature values under the rotating speed $1000 \mathrm{rpm}$ and 3000 rpm of PM turntable in Fig. 8 are very different. This is because the heat loss caused by AMF is basically unchanged at the low rotating speed of PM turntable (less than $3000 \mathrm{rpm}$ ) during the $10-4-\mathrm{mm}$ process. And only when the speed is over $3000 \mathrm{rpm}$, can the temperature rise be obviously caused inside the bulk HTS, which is verified by the temperature curves under rotating speed from $3000 \mathrm{rpm}$ to $5000 \mathrm{rpm}$. By contrast, the temperature rise of the bulk HTS is relatively high even at low speed during the $20-4-\mathrm{mm}$ process due to the violent flux motion. And at $9000 \mathrm{rpm}$, the internal temperature rise of the bulk HTS reaches $160 \mathrm{~K}$.

According to the above analysis, higher $\mathrm{CH}$ means more flux captured at the same $\mathrm{WH}$ and also means greater loss in the bulk HTS.

\subsection{Influence of WH on temperature characteristics}

We choose the $\mathrm{CH}$ of $20 \mathrm{~mm}$ to study the maximum loss of the bulk HTS, in which case it is easier to find the effect of WH on the internal temperature rise of the bulk HTS. Figures 9, 10, and 11 show the variation of temperature with time under the different rotating speeds of the PM turntable in the cases that $\mathrm{CH}$ is $20 \mathrm{~mm}$ and the $\mathrm{WH}$ is 2,4 , 6 , and $8 \mathrm{~mm}$, respectively.

From Figs. 9, 10, and 11, we can see that the lower the $\mathrm{WH}$, the higher the temperature. This can be explained as follows:

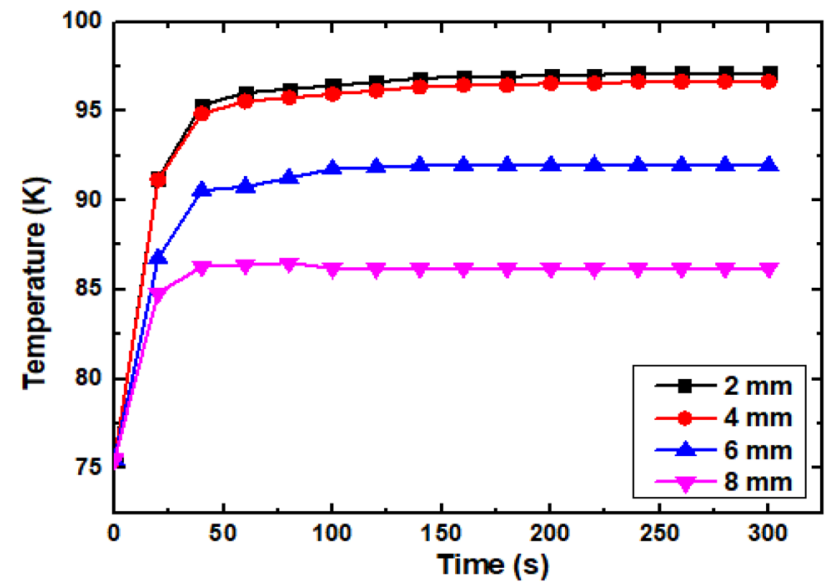

Fig. 9 Variation of temperature with time under rotating speed of the turntable $1000 \mathrm{rpm}$ in the cases that the $\mathrm{CH}$ is $20 \mathrm{~mm}$ and the $\mathrm{WH}$ is $2,4,6$, and $8 \mathrm{~mm}$, respectively

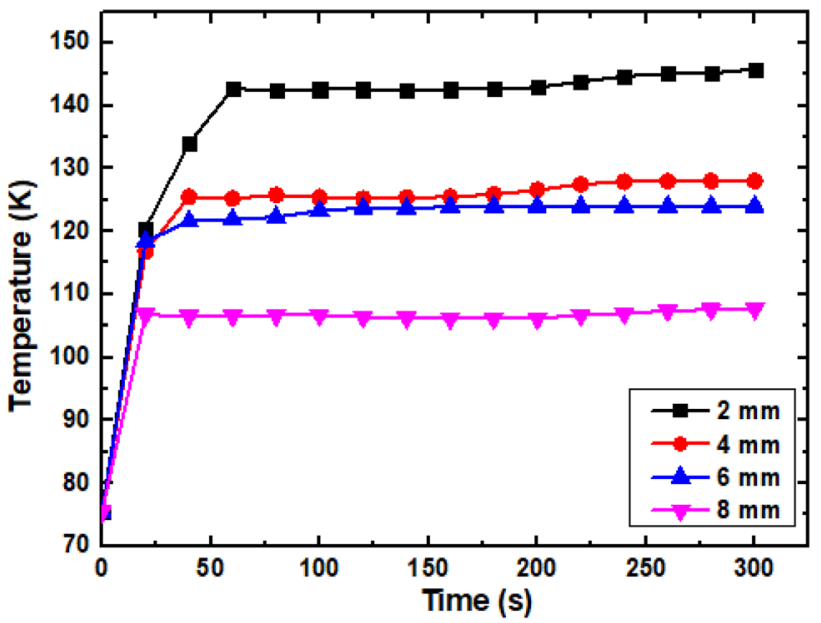

Fig. 10 Variation of temperature with time under rotating speed of the turntable $5000 \mathrm{rpm}$ in the cases that the $\mathrm{CH}$ is $20 \mathrm{~mm}$ and the $\mathrm{WH}$ is $2,4,6$, and $8 \mathrm{~mm}$, respectively

The amplitude of the external MFD affects the induced current of bulk HTS, which affects the penetration depth of bulk HTS. The penetration depth of bulk HTS under the AMF can be expressed as

$r_{\mathrm{ac}}=\frac{H_{\mathrm{AC}}}{\mu_{0} J_{\mathrm{C}}}$

where $\mu_{0}$ is the vacuum permeability, $J_{C}$ is the critical current density, and $H_{\mathrm{AC}}$ is the amplitude of the external MFD. The hysteresis loss caused by the external MFD in the unit cycle of the bulk penetration can be expressed as

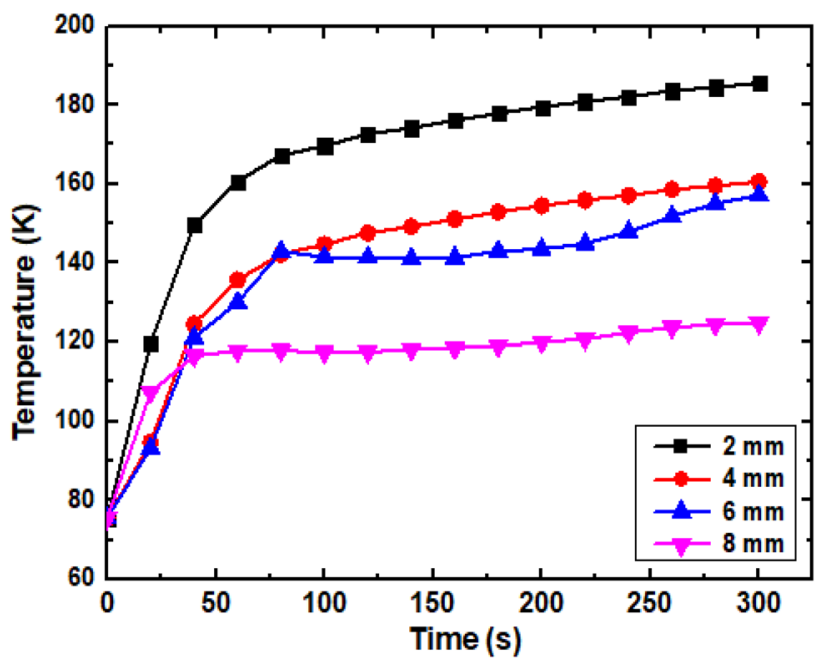

Fig. 11 Variation of temperature with time under rotating speed of the turntable $9000 \mathrm{rpm}$ in the cases that the $\mathrm{CH}$ is $20 \mathrm{~mm}$ and the $\mathrm{WH}$ is $2,4,6$, and $8 \mathrm{~mm}$, respectively 
$w=\frac{2 \mu_{0} H_{\mathrm{AC}}^{3}}{3 J_{\mathrm{C}} r_{\mathrm{ac}}}$

According to (2), we know that the hysteresis loss has a three-power relationship with $H_{\mathrm{AC}}$ and increases with $H_{\mathrm{AC}}$. In Figs. 5 and 6, we can see that the lower the $\mathrm{WH}$, the higher the MFD. Therefore, the temperature of the bulk HTS is higher under the lower WH due to the more hysteresis loss under the higher MFD.

By comparing Figs. 9, 10, and 11, we further noticed that when the rotating speed is low (for instance, $1000 \mathrm{rpm}$ ), the temperature rise curves under $2 \mathrm{~mm}$ and $4 \mathrm{~mm}$ almost coincide, as shown in Fig. 9. However, when the speed is $5000 \mathrm{rpm}$ and $9000 \mathrm{rpm}$, as shown in Figs. 10 and 11, respectively, the temperature rise at $2 \mathrm{~mm}$ is largest, and the temperature rise under WHs of $4 \mathrm{~mm}$ and $6 \mathrm{~mm}$ is almost same. In the future application of bulk HTS in an AMF, we do not suggest choosing the $\mathrm{WH}$ of $2 \mathrm{~mm}$ because the internal loss of the bulk HTS is too high under this case.

\subsection{Influence of rotating speed of PM turntable on temperature characteristics}

Figure 12 shows the temperature rise curves of the rotating speeds under $1000 \mathrm{rpm}, 3000 \mathrm{rpm}, 5000 \mathrm{rpm}$, $7000 \mathrm{rpm}$, and $9000 \mathrm{rpm}$ at $20-2 \mathrm{~mm}$. It can be seen that the temperature rises gradually with the increase in rotating speed. The highest temperature reaches $185 \mathrm{~K}$ when the rotating speed is $9000 \mathrm{rpm}$.

Moreover, it can be seen that the temperature rises higher as the speed increases, which can also be concluded from Figs. 7 and 8. We also found that under

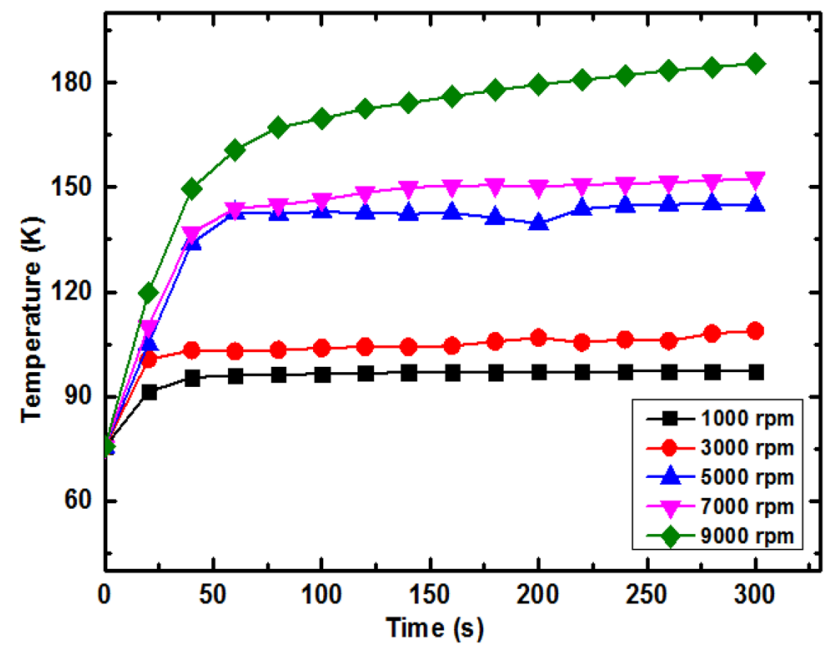

Fig. 12 Variation of temperature with time under rotating speed of PM turntable $1000 \mathrm{rpm}, 3000 \mathrm{rpm}, 5000 \mathrm{rpm}, 7000 \mathrm{rpm}$, and $9000 \mathrm{rpm}$ in the case that the $\mathrm{CH}$ is $20 \mathrm{~mm}$ and the WH is $2 \mathrm{~mm}$ rotating speed of $1000 \mathrm{rpm}$ and $3000 \mathrm{rpm}$, the temperature rise of the bulk HTS reaches the saturation point at the time of $50 \mathrm{~s}$ and there is little change after that. But at the speed of $9000 \mathrm{rpm}$, the temperature rise does not reach a stable value even at the end of $300 \mathrm{~s}$, which is caused by the AMF at high speed.

The analysis shows that at the condition of $20-2 \mathrm{~mm}$ the temperature rise under $1000 \mathrm{rpm}$ is similar to that under $3000 \mathrm{rpm}$. The maximum value of temperature rise under $5000 \mathrm{rpm}$ is $36 \mathrm{~K}$ higher than that under $3000 \mathrm{rpm}$ (about $33.1 \%$ increased), and the maximum value of temperature rise under $7000 \mathrm{rpm}$ is $7.7 \mathrm{~K}$ higher than that under $5000 \mathrm{rpm}$ (about $5.3 \%$ increased). However, the temperature rise under $9000 \mathrm{rpm}$ is extremely intense, reaching $185 \mathrm{~K}$, which is $33.1 \mathrm{~K}$ higher than the maximum value under $7000 \mathrm{rpm}$ (about $30.2 \%$ increased). In the range of 0-7000 rpm, the temperature rise tends to be saturated, but after more than 7000 rpm, there will be an obvious temperature rise.

Here, I would like to explain a common phenomenon in Figs. $7,8,9,10,11$, and 12. When the temperature of the bulk HTS increases above $92 \mathrm{~K}$ (the critical temperature of the bulk HTS), the temperature will continue to rise. Because the MFD under the bottom of the bulk HTS is different when the bulk HTS decreases from $\mathrm{CH}$ to $\mathrm{WH}$, the magnetic flux captured by each part of the bulk is different. We can see from Figs. 5 and 6 that the MFD of every point above the surface of the PMs is different. When exposed to AMF, the part with more magnetic flux captured becomes normal, while the part with less magnetic flux captured is still in superconducting state and the temperature still keeps rising. The sensor only measures the temperature of a certain position in the bulk HTS, not the temperature of the whole bulk HTS. The loss of each part of the bulk depends on the magnitude of the magnetic field. On the other hand, because of the poor thermal conductivity of the bulk, the heat cannot be emitted well. This is why after the numerical value of the temperature sensor increases above $92 \mathrm{~K}$, there are still loss and temperature rise.

\section{Conclusion}

The temperature characteristics of bulk HTS exposed to AMF have been studied, and results have been discussed. Firstly, at the same $\mathrm{CH}$, lower WH means greater loss. The loss of WH $2 \mathrm{~mm}$ is significantly higher than that of $4 \mathrm{~mm}$, $6 \mathrm{~mm}$ and $8 \mathrm{~mm}$. Secondly, temperature inside the bulk HTS tends to be saturated with the increase in rotating speed. However, when it reaches $9000 \mathrm{rpm}$ and there will still be a distinct temperature rise, which should be paid attention to in the practical application of bulk HTS. At the next stage, we will use slitting to reduce the eddy current 
of the bulk HTS and establish a simulation model to analyze temperature rise inside the bulk HTS exposed to AMF. Besides, we will optimize the system structure to test the effect of temperature on the thermal stress of the system.

Contributions of this paper include:

- A high-speed test device is designed and manufactured to generate a high-frequency and high-gradient MFD, which would be useful in research on rotating HTS levitation system (bearing) when the PMs break locally or periodically.

- These data provide theoretical references to future superconducting Maglev-evacuated tube transport system under extremely harsh conditions, which are also useful for the HTS synchronous motor. There is a very high reference value to analyze the loss of the bulk HTS.

Funding This study was funded by the "Research on New Superconducting Flywheel Energy Storage Technology and Superconducting Flywheel Storage technology Based on Superconducting Magnetic Constraints" (No. 6141B08110404) and "Chinese National Natural Science Foundation" (No. 51407015).

\section{Compliance with ethical standards}

Conflict of interest The authors declare that they have no conflict of interest.

\section{References}

1. Zhao Y, Ji C, Yang Y, Zhao L, Zhou D, Zhang Y (2019) Dynamical behavior of HTS maglev system over a NdFeB guideway with a fluctuant field distribution. IEEE Trans Appl Supercond 29(2):1-6

2. Zhou D et al (2018) High-Tc superconducting maglev prototype vehicle running at $160 \mathrm{~km} / \mathrm{h}$ in an evacuated circular track. IEEE Trans Appl Supercond 28(4):1-4

3. Deng Z et al (2016) A high-temperature superconducting maglev ring test line developed in Chengdu, China. IEEE Trans Appl Supercond 26(6):1-8

4. Werfel FN et al (2016) Experiments of superconducting maglev ground transportation. IEEE Trans Appl Supercond 26(3):1-5

5. Wang JS, Wang SY, Zeng Y et al (2002) The first man-loading high temperature superconducting maglev test vehicle in the world. Phys C Supercond 378381(1):809-814

6. Terao Y, Sekino M, Ohsaki H (2010) Design study of linear synchronous motors using superconducting coils and bulks. In: Proceedings of IPEC, pp 1760-1765

7. Sekiguchi D et al (2012) Trial test of fully HTS induction/synchronous machine for next generation electric vehicle. IEEE Trans Appl Supercond 22(3):5200904
8. Xun JJ, Hai ZL, Guang GY, Guo ZJ, Grantham C, Sorrell CC (2012) High-temperature superconducting linear synchronous motors integrated with HTS magnetic levitation components. IEEE Trans Appl Supercond 22(5):5202617-5202620

9. Li W, Chau KT, Ching TW, Wang Y, Chen M (2015) Design of a high-speed superconducting bearingless machine for flywheel energy storage systems. IEEE Trans Appl Supercond 25(3):1-4

10. Mukoyama $S$ et al (2017) Development of superconducting magnetic bearing for $300 \mathrm{~kW}$ flywheel energy storage system. IEEE Trans Appl Supercond 27(4):1-4

11. Xu K, Wu D, Jiao YL, Zheng MH (2016) A fully superconducting bearing system for flywheel applications. Supercond Sci Technol 29(6):064001

12. Wang S, Zheng J, Song $H$, Wang $X$, Wang J (2005) Experiment and numerical calculation of high temperature superconducting Maglev. IEEE Trans Appl Supercond 15(2):2277-2280

13. Wang JS, Wang SY, Ren Z, Zhu M, Jiang H, Tang Q (2001) Levitation force of a $\mathrm{YBaCuO}$ bulk high temperature superconductor over a NdFeB railway. IEEE Trans Appl Supercond 11(1):1801-1804

14. Park YG et al (2015) Experimental analysis on initial current decay characteristics of persistent-mode HTS coil by external alternating magnetic field. IEEE Trans Appl Supercond 25(3):1-4

15. Geng J, Zhang H, Li C, Zhang X, Shen B, Coombs TA (2017) Angular dependence of direct current decay in a closed YBCO doublepancake coil under external AC magnetic field and reduction by magnetic shielding. Supercond Sci Technol 30(3):035022

16. Geng J, Coombs T (2015) Mechanism of a high- $T_{C}$ superconducting flux pump: using alternating magnetic field to trigger flux flow. Appl Phys Lett 107(14):142601

17. Li J et al (2010) Experimental study of the electromagnetic forces of a HTS bulk magnet subjected to sinusoidal traveling magnetic field. IEEE Trans Appl Supercond 20(3):929-932

18. Ogawa J, Nakayama H, Odaka S, Tsukamoto O (2005) AC loss characteristics of YBCO coated conductors carrying transport currents in external AC magnetic fields. Cryogenics 45:23-27

19. Ichiki Y, Ohsaki H (2004) Numerical analysis of AC losses in YBCO coated conductor in external magnetic field. Phys $C$ 412414:1015-1020

20. Xu K, Wu D-J, Jiao YL, Zheng MH (2016) A fully superconducting bearing system for flywheel applications. Supercond Sci Technol 29(6):64001

21. Deng Z et al (2017) A high-temperature superconducting Maglev-evacuated tube transport (HTS Maglev-ETT) test system. IEEE Trans Appl Supercond 27(6):1-8

22. Huang Z, Zhang M, Wang W, Coombs TA (2014) Trial test of a bulk-type fully HTS synchronous motor. IEEE Trans Appl Supercond 24(3):1-3

23. Zheng X, Yang Y (2007) Transition cooling height of high-temperature superconductor levitation system. IEEE Trans Appl Supercond 17(4):3862-3866

Publisher's Note Springer Nature remains neutral with regard to jurisdictional claims in published maps and institutional affiliations. 